\title{
Physician burnout and professional satisfaction in orthopedic surgeons during the COVID-19 Pandemic
}

\author{
Alexander L. Lazarides*, Elshaday S. Belay, Albert T. Anastasio, \\ Chad E. Cook and Oke A. Anakwenze \\ Department of Orthopaedic Surgery, Duke University Medical Center, Durham, NC, USA
}

Received 3 December 2020

Accepted 7 January 2021

\begin{abstract}
.
BACKGROUND: Burnout and professional satisfaction is an often an overlooked component for healthcare outcomes; the COVID-19 pandemic represents an unprecedented stressor that could contribute to higher levels of burnout.

OBJECTIVES: Our primary objective was to evaluate the association of a battery of fulfillment, job satisfaction change, COVID-19 concerns, and coping measures. Our secondary objective was to determine whether the fulfillment and coping measures differed by gender and by experience levels among a battery of physician specialties.

METHODS: The study was a purposive sample of convenience. Study participants included all trainees and attending orthopedic surgeons from our academic institution; all participants were invited to complete a survey built around a validated measure of professional fulfillment aimed at assessing response to acute change and stressors. We performed univariate statistics and a matrix correlational analysis to correlate different survey domains with variables of interest.

RESULTS: The survey was sent electronically to 138 individuals; 63 surveys were completed $($ response rate $=45.7 \%)$. Twenty-seven (42.8\%) individuals met the threshold criteria for fulfillment whereas 10 (15.9\%) met the threshold for burnout. We found that surgeon perspectives on COVID-19 were not associated with burnout or professional fulfillment. Burnout was inversely associated with professional fulfillment $(R=-0.35)$. Support seeking was noted to be correlated with professional fulfillment $(R=0.37)$.

CONCLUSIONS: Stressors related to COVID-19 pandemic were not correlated with physician burnout and fulfillment. This held true even when stratifying by gender and by attending vs. trainee. Continued efforts should be implemented to protect against physician burnout and ensure professional fulfillment for Orthopedic surgeons.
\end{abstract}

Keywords: Professional fulfillment, orthopaedic surgery, support strategies, coping mechanisms, burnout

\section{Background}

Burnout and professional satisfaction are an often overlooked component for healthcare outcomes. Physician career fulfillment has a positive correlation with patient satisfaction, patient -physician relationships, and compliance with treatment $[1,2]$. Professional satisfaction has also been shown to protective against physician burnout [3]. Unfortunately,

*Address for correspondence: Alexander Lazarides MD, Department of Orthopaedic Surgery, 311 Trent Dr, Durham, NC 27710, USA. E-mail: Alexander.lazarides@duke.edu. physician burnout continues to be a growing burden in the healthcare field. In the United States, more than half of all physicians experience symptoms of burnout resulting in a disproportionately higher prevalence among physicians compared to the general workforce [4].

Burnout is defined by a combination of emotional exhaustion, depersonalization and decreased sense of accomplishment due to stress. However, the manifestation of burnout is particularly underreported in orthopedic surgery. In a 2016 systematic review, only $15 \%$ of all studies on surgeon burnout have examined the topic in orthopedic surgeons and trainees. 
The consequences of burnout are numerous including committing medical errors, depression and thoughts of self-harm [5, 6]. Currently, the origin of burnout among surgeons is thought to be multifactorial with risk factors including gender, work-life balance and practice setting $[7,8]$.

In 2019, the arrival of COVID-19 has been shown to increase stressors for physicians [9-11]. Studies from China demonstrate a significant rise in rates of depression and anxiety for health care workers during the current pandemic [12]. In the United States, there has been a significant re-direction of physicians from a number of sub-specialties to the frontlines in this pandemic. Additionally, as a sub-specialty with a large number of elective procedures, both state and federal mandates regarding elective procedures have directly impacted the practice of several orthopedic providers [13]. The changes in education with restricted in-person activity and cancelled large group events have also altered the educational experience for orthopedic trainees [14]. With these changes, the short and long-term effect of the COVID-19 pandemic on risks of burnout in orthopedic surgeons is unknown.

The purpose of this study is twofold. Our primary objective was to evaluate the association of a battery of fulfillment, job satisfaction change, COVID-19 concerns, and coping measures. Our secondary objective was to determine whether the fulfillment and coping measures differed by gender and by experience levels among a battery of physician specialties. We hypothesized that higher rates of burnout and decreased professional fulfillment would be associated with increased concerns regarding COVID-19.

\section{Methods}

\subsection{Study design}

This study received institutional review board approval. The study used a cross sectional, electronic survey design (E-survey). We followed the "Improving the Quality of Web Surveys: The Checklist for Reporting Results of Internet E-Surveys (CHERRIES)" checklist to improve reporting standards.

\subsection{Participants}

The study was a purposive sample of convenience. Study participants included all trainees and attending orthopedic surgeons from our academic institution.
All faculty, fellows and residents were invited to participate via email to complete an online survey. Data was collected from April 202020 through April 30 2020. The survey was housed and built in Red Cap. Data was collected and deposited automatically in a secure Red Cap database in an anonymous manner. All participation was voluntary and each entry was linked to the email address invited to ensure unique site visitation and preventing multiple entries from the same individual. There was no incentive offered for participation. Respondents were allowed to check and change their answers prior to completion; a completeness check was performed automatically for each survey. Only complete surveys were included, and there were no incomplete surveys excluded.

\subsection{Survey questionnaires (Supplement)}

Two formal questionnaires were used to identify the full breath of survey recipient response. The first was the Professional Fulfillment Index, which includes 16 items and two subscales (fulfillment and burnout), was included to capture sensitivity to change in professional fulfilment and burnout that might be attributed to influences affecting physician happiness and mental health, particularly as it relates to COVID-19 [15]. The Professional Fulfillment Index (PFI) has been used previously within the literature and is a validated short form measure of burnout [15]. This measure has been validated as a surrogate for the Maslach Burnout Inventory (MBI), a burnout measure, as well as the World Health Organization's WHOQOL-BREF, which is built to assess quality of life. While the MBI is considered the standard for assessing burnout, it is not well suited to assess changes due to external factors such as from COVID-19 [16]. In contrast, the PFI as better suited for assessing changes in burnout and fulfillment related to acute stressors; it has previously been validated and utilized in physicians. For each item, Likert scores represented ordinal choices for fulfillment (positively oriented) and burnout (negatively oriented). Previous work has defined two thresholds for fulfillment and burnout. Individuals scoring professional fulfillment values of $>18$ points from six dedicated questions regarding work satisfaction, control and meaningfulness were coded at "fulfilled". Individuals scoring coping burnout scores of $>14$ points from the remaining ten items were coded as "burned out".

The second formal questionnaire, consisting of three questions was designed to evaluate the 
prevalence and association between specific at-work coping strategies and professional fulfillment and burnout. Three specific coping strategies were evaluated; problem solving (planning), support seeking (collegial engagement) and mental breaks (mental strategies). These coping strategies have been previously studied and noted to be associated with burnout symptoms or lack thereof [17]. Each item was coded with a four choice Likert response, with higher values reflecting stronger coping strategies.

Several novel questions assessing future practice outlook and most significant COVID-19 concerns were incorporated in the survey as well. The role of the physician outlook (positive and negative) have been shown to be contributory factors to burnout. These questions included three specific COVID-19 related questions associated with threats of financial, health, and practice pattern disruption, and one question related to job satisfaction. Each COVID-19 question was coded with a Likert choice, whereas the job satisfaction question was recoded as Less or Same/Better after collapsing the three choices into two.

\subsection{Purposive sampling estimation}

Our study goal was to sample (purposively) all orthopedic surgeons and trainees in our academic healthcare system. This included a total of 138 surgeons (80 faculty and 58 trainees).

\subsection{Statistical analysis}

Subject characteristics were described by frequencies, means and percentages. For our primary objective, we analyzed associations between the dichotomized values for professional fulfillment and burnout, three coping questions, questions associated with concerns with the COVID-19 virus's influence of practice and change in job satisfaction (dichotomized). To analyze this, we used a Gamma statistic when evaluating ordinal-to-ordinal measures, a phi coefficient when measuring nominal to nominal, and a chi-square contingency correlation when comparing the ordinal to nominal values. Correlational values ranged from -1 to 0 to +1 , which values closer to -1 and +1 representing stronger inverse or position associations.

For our secondary objective, we measured differences in our three fulfillment domains by gender and by training level (i.e., trainees versus experience physicians). Since the three summated domains of the professional fulfillment index were not normally distributed, we used a Mann Whitney U for ordinal analyses and a chi square for our nominal measures (fulfillment and burnout), using a $p$ value of 0.05 to denote statistical significance.

\section{Results}

The survey was sent to 138 individuals and 63 were returned (response rate $=45.7 \%$ ). A majority $(51 \%)$ of the respondents were 25 to 40 years of age, with 4 over the age of 70 . Respondents included trainees $(\mathrm{N}=22)$ and experienced $(\mathrm{N}=41)$ orthopedic surgeons. Approximately $16 \%$ of practicing surgeons had 15 to 25 years of experience whereas $25 \%$ had greater than 25 years of experiences. Nine (14.2\%) respondents were female.

\subsection{Burnout and professional fulfilment during COVID-19}

In our sample of 63 orthopedic surgeons, we noted mean professional fulfillment and burnout scores of $14.7(\mathrm{SD}=6.4)$ and $7.8(\mathrm{SD}=5.8)$, respectively. Twenty-seven $(42.8 \%)$ individuals met the threshold criteria for fulfillment whereas $10(15.9 \%)$ met the threshold for burnout. There were no differences noted in the incidence of significant burnout or lack of professional fulfilment based on gender or trainee status (Table 1).

\subsection{Factors associated with burnout and professional fulfilment during COVID-19}

For our objective of analyzing the associations among fulfillment, burnout, job satisfaction and COVID19 concerns, we noted that evidence of burnout

Table 1

Differences in incidence of significant burnout or lack of professional fulfillment

\begin{tabular}{llcc}
\hline & Category & Mean (SD) & $P$ value \\
\hline Gender & & & \\
Burned out & Male & $9(7 \%)$ & 0.67 \\
& Female & $1(11 \%)$ & \\
$\begin{array}{l}\text { Professionally } \\
\quad \text { uulfilled }\end{array}$ & Male & $17(41 \%)$ & 0.41 \\
$\begin{array}{l}\text { Training level } \\
\text { Burned out }\end{array}$ & Female & $10(45 \%)$ & \\
& Trainee & $2(9 \%)$ & 0.49 \\
Professionally & Experienced & $8(20 \%)$ & \\
$\quad$ Tulfilled & Experienced & $10(45 \%)$ & 0.76 \\
\hline
\end{tabular}


was inversely associated with professional fulfillment $(R=-0.35)$. Burnout subgroup analysis revealed a correlation between evidence of work exhaustion and depersonalization $(R=-0.48)$. Notably, surgeon post COVID-19 outlook was not associated with burnout or professional fulfillment.

Table 2

Strength of different coping strategies based on gender and practice status

\begin{tabular}{lllc}
\hline & Category & Mean (SD) & $P$ value \\
\hline Gender & & & \\
Creating plans Coping & Male & $2.5(0.9)$ & 0.36 \\
& Female & $2.8(0.6)$ & \\
Turn to & Male & $2.2(0.8)$ & 0.38 \\
colleagues Coping & Female & $2.4(1.0)$ & \\
Mental breaks & Male & $2.1(0.8)$ & 0.39 \\
& Female & $2.3(0.8)$ & \\
Training level & & & \\
Creating plans Coping & Trainee & $2.4(0.9)$ & 0.37 \\
& Experienced & $2.6(0.8)$ & \\
Turn to colleagues & Trainee & $2.2(1.0)$ & 0.89 \\
& Experienced & $2.2(0.9)$ & \\
Mental breaks & Trainee & $2.3(0.9)$ & 0.15 \\
& Experienced & $2.0(0.7)$ & \\
\hline
\end{tabular}

\subsection{Differences in coping strategies during COVID-19}

Three coping strategies were analyzed in this study; problem solving, support seeking and mental breaks. Of these, support seeking (turning to a colleague for support) was noted to be correlated with professional fulfillment $(R=0.37)$. We noted that coping measures were inter-related and associated with one another. No differences were noted in the strength of any coping measure based on gender or trainee and practicing status (Table 2). The above results are summarized in the correlation matrix (Fig. 1).

\section{Discussion}

Physician burnout remains an increasingly challenging problem facing healthcare. With the arrival of the COVID-19 pandemic, there are concerns that the stressors contributing to physician burnout may only be magnified. One might assume this would particularly be the case in specialties with a heavy elective component of their practice, such as Orthopaedics,

\begin{tabular}{|c|c|c|c|c|c|c|c|c|c|}
\hline & $\begin{array}{c}\text { Financial } \\
\text { Concerns } \\
\text { about } \\
\text { COVID }\end{array}$ & $\begin{array}{l}\text { Health } \\
\text { Concerns } \\
\text { about } \\
\text { COVID }\end{array}$ & $\begin{array}{l}\text { Practice } \\
\text { Pattern } \\
\text { Concerns } \\
\text { COVID }\end{array}$ & $\begin{array}{c}\text { Burned } \\
\text { Out }\end{array}$ & $\begin{array}{c}\text { Profession- } \\
\text { ally } \\
\text { Fulfilled }\end{array}$ & $\begin{array}{l}\text { Change in } \\
\text { Job } \\
\text { Satisfaction }\end{array}$ & $\begin{array}{l}\text { Coping- } \\
\text { Creating } \\
\text { Plans }\end{array}$ & $\begin{array}{c}\text { Coping- } \\
\text { Turn to } \\
\text { Colleagues }\end{array}$ & $\begin{array}{c}\text { Coping- } \\
\text { Take } \\
\text { Mental } \\
\text { Breaks }\end{array}$ \\
\hline $\begin{array}{l}\text { Financial } \\
\text { Concerns } \\
\text { about COVID }\end{array}$ & 1.0 & & & & & & & & \\
\hline $\begin{array}{l}\text { Health } \\
\text { Concerns } \\
\text { about COVID }\end{array}$ & $.25 *$ & 1.0 & & & & & & & \\
\hline $\begin{array}{l}\text { Practice } \\
\text { Pattern } \\
\text { Concerns } \\
\text { COVID }\end{array}$ & $.27 *$ & -.02 & 1.0 & & & & & & \\
\hline Burned Out & .07 & .06 & .03 & 1.0 & & & & & \\
\hline $\begin{array}{l}\text { Professionally } \\
\text { Fulfilled }\end{array}$ & .05 & .08 & .00 & $-.35 * *$ & 1.0 & & & & \\
\hline $\begin{array}{l}\text { Change in Job } \\
\text { Satisfaction }\end{array}$ & .17 & -.08 & .04 & .27 & .25 & 1.0 & & & \\
\hline $\begin{array}{l}\text { Coping- } \\
\text { Creating Plans }\end{array}$ & .02 & .03 & .18 & .32 & .31 & .16 & 1.0 & & \\
\hline $\begin{array}{l}\text { Coping- Turn } \\
\text { to Colleagues }\end{array}$ & .04 & .09 & .03 & .24 & $.37 *$ & -.21 & $.66^{* *}$ & 1.0 & \\
\hline $\begin{array}{l}\text { Coping-Take } \\
\text { Mental Breaks }\end{array}$ & .04 & .11 & .01 & .12 & .17 & -.10 & $.38 *$ & $.55^{* *}$ & 1.0 \\
\hline
\end{tabular}

* Correlation is significant at the 0.05 level (2-tailed). ${ }^{* *}$ Signifies significance at 0.01 level.

Fig. 1. Correlation matrix of associations for fulfillment index, coping measures, concerns about COVID-19 and job satisfaction changes. ${ }^{*}$ Correlation is significant at the 0.05 level (2-tailed). ${ }^{* *}$ Signifies significance at 0.01 level. 
that have seen their patient care volume disproportionately affected. This study aimed to determine the magnitude of physician burnout and professional fulfillment during the COVID-19 pandemic amongst an orthopaedic surgery department in an academic tertiary care referral hospital. While we noted a high prevalence of concern regarding post COVID-19 outlook, orthopedic surgeons were found to have a low incidence (11\%) of burnout. Unfortunately, only $43 \%$ of orthopedic surgeons were noted to be professionally fulfilled during the COVID-19 pandemic. In addition, we did not note a difference in the level of burnout between trainees and faculty which is contrary to prior reports, nor when stratifying by gender. We noted that orthopedic surgeons with higher levels of professional fulfillment were less likely to have high levels of depersonalization and work exhaustion, both the primary indicators of clinical burnout [18]. Finally, we found that surgeons with better coping mechanisms, particularly seeking out support from colleagues, had higher levels of professional fulfilment.

Despite the relative recency of the COVID-19 global spread, a growing body of literature exists delineating mental health and burnout effects of the pandemic on health care workers [11, 19-24]. ElHage et al. performed a narrative review of work pertaining to mental health implications of previous epidemics, the 2003 SARS-CoV-1 outbreak and the 2009 H1N1 outbreak [19]. They determined that when compared to these previous epidemics, the COVID-19 pandemic may have characteristics particularly detrimental to health care workers responding to the pandemic, given rapid spread and disease symptom severity [19]. Furthermore, these authors cite organizational factors, such as potential shortages of ventilators and intensive care unit beds, depletion of personal protection equipment, and significant change in daily life outside of the hospital given quarantine restrictions as being other potential exacerbating factors for burnout amongst health care workers in the COVID-19 era [19]. In addition, practitioners in elective fields such as orthopedic surgery, have seen a rapid decline in their practice volume due to the mandatory restrictions. Further, the nature of patient engagement, while lower in incidence, has seen significant transformation from mostly traditional live patient encounters to virtual telehealth platforms. The psychological effects of these sudden changes is critically important in order to plan for future crises and to ensure that necessary resources are available.
In Pakistan, Rana et. al. report growing mental health concerns among health care workers responding to the COVID-19 outbreak [23]. These authors call for the development of a psychological crisis intervention model (PCIM), where integrated teams of physicians, psychiatrists, psychologists/mental health practitioners, and social workers could provide early psychological intervention to health care workers at high risk for burnout [23]. In contrast to these results, Tan et.al. in Singapore report lower Depression, Anxiety, and Stress Scales (DASS-21) and Impact of Events Scale-Revised (IES-R) instrument scores in COVID-19 era health care workers as compared to scores obtained during the SARS pandemic [24]. Reported rates of PTSD were three times lower than those reporting during the SARS experience [24]. Furthermore, rates of depression and anxiety in non-health care workers in Singapore are higher than those of health care workers [24]. These findings are corroborated by Li et. al. in China [21]. Tan et. al. cite less intensive training on personal protective equipment and infection control measures as well as less up-to-date medical information on the outbreak as potential rationale for why non-health care workers may experience greater mental health consequences than health care workers during the COVID-19 pandemic [24]. A growing body of literature pertains to declining mental health of the general public in the COVID-19 era, and health care workers should be made aware of these trends and respond accordingly [25-29]. In our study, we noted a $36.5 \%$ rate of health concerns amongst orthopedic surgeons. We did not note this to be associated with professional fulfillment and burnout. This may indicate a favorable measure of resilience amongst orthopedic surgeons, trust in screening practices or in other preventative measures. However, it may also represent an aspect of physician wellbeing that is not related to burnout. While this was not the purpose of our study, we believe that it may be worth studying in future.

Our study found no differences in burnout between attending physicians and residents nor by gender. These finding are in discordance with other findings in the literature. In a systematic review by Pulcrano et al., residents were at risk for burnout than attendings; the areas in which this discrepancy was most notable included orthopedic surgery [30]. Interestingly, gender did not have a clear impact. These prior findings are not surprising when considering factors such as longer work hours, lack of social support and mentors, financial debt and others. The COVID-19 crisis presents a unique challenge for both trainees 
and attending as it pertains to professional fulfillment and burnout. It is possible, though, that reduced work hours during the pandemic may have played a role in the lack of significant differences noted between our trainees and the faculty.

Our study reaffirms the importance of effective coping strategies and resiliency for avoiding burnout. Coping encompasses both active and passive responses to stressors or threats to help mitigate those stressors or threats [31, 32]. We found that physicians who took an active role in creating plans to improve practice conditions felt greater professional fulfillment and less depersonalization. The importance of building resiliency and coping strategies have been well borne out in the literature. In interviews from 200 physicians, Zwack et al. found that physicians with more effective coping strategies had improved resiliency; in concert with this, they found that resiliency was central to the concept of physician well-being [33]. Sargent et al. surveyed a large cohort of Orthopedic residents and attendings and identified several protective factors for building resiliency and preventing burnout [34]. For residents, protective factors included having control over one's time, taking mental breaks, mentorship and support. These factors were similar for attendings. While our study did not find an association between support and mental breaks and fulfillment, the association between making plans is not surprising. Denial, cynicism and avoidance are negative coping strategies [32]; Firth-Cozens et al showed that, in medical students, utilization of these coping strategies were not effective in preventing burnout and coping with stress [35]. It stands to reason that physicians more willing to acknowledge challenges and make plans to help navigate those stressors and challenges would be better equipped to cope with stress and have greater resiliency. In response to COVID-19 stressors, seeking support from colleagues and taking mental breaks was not associated with decreased signs of burnout. This may indicate a different set of stressors unique to the COVID-19 crisis as opposed to the traditional stressors associated with burnout.

While this study provides insight into the relationship between the effect of a pandemic on physician burnout, it is not without limitations. As this was a cross- sectional study, there are several inherent considerations. The first limitation to consider with this study is that all data collected was by physician self-report. As such, there is the inherent possibility of bias, particularly selection and recall bias. To the former, it is possible that the physicians most experiencing burnout and the effects of the COVID19 pandemic would be least inclined to participate in such a survey, thus underestimating the correlation between a pandemic and the COVID-19 pandemic. At the other end of the spectrum, it is also possible that the physicians who have maintained the most consistent volume practice may not have had the time to participate in such a study, again falsely skewing the results. As for recall bias, it is possible that physicians had a false estimate of their experience over the questionnaire time frame, though this is would seem to be less likely.

It is also certainly worth noting that these results may not be generalizable to orthopaedic surgeons as a whole. While the entire US has been affected by the pandemic, it is true that certain regions have been affected to a larger degree than others. In response to the COVID-19 lock down at our institution, orthopaedic residents where split into three "platoons," with each platoon working for one straight week, followed by two weeks of quarantine at home. Elective surgery was largely halted; cases that did proceed were ultimately subject to review for suitability to proceed. "On-week" residents largely covered emergency department consults, orthopaedic trauma cases, and emergent joint and soft tissue infection irrigation and debridement. Unlike other institutions, Orthopaedic residents were not required to undergo reallocation staffing of intensive care units to manage patients with COVID-19 due to provider shortages. It is possible that the stressors felt at our academic institution in the face of a pandemic are not the same as institutions in major metropolitan areas; similarly, it is possible that private and community practices are affected in a different manner than academic centers, which may have a greater reserve for maintaining employment and withstanding a financial and patient care volume hit.

Finally, it is important to note that this study can only imply association and not causality. As such, while it stands to reason that certain coping strategies would lend themselves to higher resiliency and lower physician burnout, this cannot be proven. Despite these limitations, we feel that this study provides an interesting snapshot into the effect of a pandemic on physician fulfillment and feelings of burnout.

\section{Conclusions}

Burnout remains an important consideration in physicians. The COVID-19 pandemic represents 
an unprecedented challenge to healthcare, forcing widespread change in practice patterns and, potentially, physician stressors. This study found that concerns about COVID-19 did not seem to be correlated with physician burnout and fulfillment. This held true even when stratifying by gender and by attending vs. trainee. Continued efforts should be implemented to protect against physician burnout and ensure professional fulfillment for orthopedic surgeons.

\section{Conflict of interest}

None of the authors report any conflicts of interest relevant to this manuscript.

\section{Funding}

No funding was received for this study.

\section{Supplementary material}

The supplementary material is available from https://dx.doi.org/10.3233/WOR-205288.

\section{References}

[1] Leigh JP, Tancredi DJ, Kravitz RL. Physician career satisfaction within specialties. BMC Health Serv Res. 2009;9:166.

[2] Olson K, Marchalik D, Farley H, Dean SM, Lawrence EC, Hamidi MS, et al. Organizational strategies to reduce physician burnout and improve professional fulfillment. Curr Probl Pediatr Adolesc Health Care. 2019;49(12):100664.

[3] Shanafelt TD, Mungo M, Schmitgen J, Storz KA, Reeves D, Hayes SN, et al. Longitudinal Study Evaluating the Association Between Physician Burnout and Changes in Professional Work Effort. Mayo Clin Proc. 2016;91(4):42231.

[4] Shanafelt TD, Sinsky C, Dyrbye LN, Trockel M, West CP. Burnout Among Physicians Compared With Individuals With a Professional or Doctoral Degree in a Field Outside of Medicine. Mayo Clin Proc. 2019;94(3):549-51.

[5] Shanafelt TD, West CP, Sinsky C, Trockel M, Tutty M, Satele DV, et al. Changes in Burnout and Satisfaction With Work-Life Integration in Physicians and the General US Working Population Between 2011 and 2017. Mayo Clin Proc. 2019;94(9):1681-94.

[6] Dimou FM, Eckelbarger D, Riall TS. Surgeon Burnout: A Systematic Review. J Am Coll Surg. 2016;222(6):1230-9.

[7] Dyrbye LN, Shanafelt TD, Balch CM, Satele D, Sloan J, Freischlag J. Relationship between work-home conflicts and burnout among American surgeons: a comparison by sex. Arch Surg. 2011;146(2):211-7.
[8] Barrack RL, Miller LS, Sotile WM, Sotile MO, Rubash HE. Effect of duty hour standards on burnout among orthopaedic surgery residents. Clin Orthop Relat Res. 2006;449: 134-7.

[9] Fessell D, Cherniss C. Coronavirus Disease 2019 (COVID19) and Beyond: Micropractices for Burnout Prevention and Emotional Wellness. J Am Coll Radiol. 2020.

[10] El Haj M, Allain P, Annweiler C, Boutoleau-Bretonniere C, Chapelet G, Gallouj K, et al. Burnout of Healthcare Workers in Acute Care Geriatric Facilities During the COVID-19 Crisis: An Online-Based Study. J Alzheimers Dis. 2020;78(2):847-52.

[11] Babamiri M, Alipour N, Heidarimoghadam R. Research on reducing burnout in health care workers in critical situations such as the COVID-19 outbreak. Work. 2020;66(2):379-80.

[12] Wu Y, Wang J, Luo C, Hu S, Lin X, Anderson AE, et al. A Comparison of Burnout Frequency Among Oncology Physicians and Nurses Working on the Frontline and Usual Wards During the COVID-19 Epidemic in Wuhan, China. J Pain Symptom Manage. 2020.

[13] O'Connor CM, Anoushiravani AA, DiCaprio MR, Healy WL, Iorio R. Economic Recovery After the COVID-19 Pandemic: Resuming Elective Orthopedic Surgery and Total Joint Arthroplasty. J Arthroplasty. 2020.

[14] Schwarzkopf R, Maher NA, Slover JD, Strauss EJ, Bosco JA, Zuckerman JD. The Response of an Orthopedic Department and Specialty Hospital at the Epicenter of a Pandemic: The NYU Langone Health Experience. J Arthroplasty. 2020.

[15] Trockel M, Bohman B, Lesure E, Hamidi MS, Welle D, Roberts L, et al. A Brief Instrument to Assess Both Burnout and Professional Fulfillment in Physicians: Reliability and Validity, Including Correlation with Self-Reported Medical Errors, in a Sample of Resident and Practicing Physicians. Acad Psychiatry. 2018;42(1):11-24

[16] Maslach C, Jackson SE, Leiter MP. Maslach Burnout Inventory Manual: Consulting Psychologists Press; 1981.

[17] Lemaire JB, Wallace JE. Not all coping strategies are created equal: a mixed methods study exploring physicians' self reported coping strategies. BMC Health Serv Res. 2010;10(1):208.

[18] West CP, Dyrbye LN, Sloan JA, Shanafelt TD. Single item measures of emotional exhaustion and depersonalization are useful for assessing burnout in medical professionals. J Gen Intern Med. 2009;24(12):1318-21.

[19] El-Hage W, Hingray C, Lemogne C, Yrondi A, Brunault P, Bienvenu T, et al. [Health professionals facing the coronavirus disease 2019 (COVID-19) pandemic: What are the mental health risks?]. Encephale. 2020.

[20] Kamerow D. Covid-19: Don't forget the impact on US family physicians. BMJ. 2020;368:m1260.

[21] Li Z, Ge J, Yang M, Feng J, Qiao M, Jiang R, et al. Vicarious traumatization in the general public, members, and nonmembers of medical teams aiding in COVID-19 control. Brain Behav Immun. 2020.

[22] Montemurro N. The emotional impact of COVID-19: From medical staff to common people. Brain Behav Immun. 2020.

[23] Rana W, Mukhtar S, Mukhtar S. Mental health of medical workers in Pakistan during the pandemic COVID-19 outbreak. Asian J Psychiatr. 2020;51:102080.

[24] Tan BYQ, Chew NWS, Lee GKH, Jing M, Goh Y, Yeo LLL, et al. Psychological Impact of the COVID-19 Pandemic on Health Care Workers in Singapore. Ann Intern Med. 2020.

[25] Chatterjee SS, Barikar CM, Mukherjee A. Impact of COVID-19 pandemic on pre-existing mental health problems. Asian J Psychiatr. 2020;51:102071. 
[26] Hiremath P, Suhas Kowshik CS, Manjunath M, Shettar M. COVID 19: Impact of lock-down on mental health and tips to overcome. Asian J Psychiatr. 2020;51:102088.

[27] Ho CS, Chee CY, Ho RC. Mental Health Strategies to Combat the Psychological Impact of COVID-19 Beyond Paranoia and Panic. Ann Acad Med Singapore. 2020;49(3):155-60.

[28] Rajkumar RP. COVID-19 and mental health: A review of the existing literature. Asian J Psychiatr. 2020;52:102066.

[29] Usher K, Durkin J, Bhullar N. The COVID-19 pandemic and mental health impacts. Int J Ment Health Nurs. 2020;29(3):315-8.

[30] Pulcrano M, Evans SR, Sosin M. Quality of Life and Burnout Rates Across Surgical Specialties: A Systematic Review. JAMA Surg. 2016;151(10):970-8.

[31] Isaksson Ro KE, Tyssen R, Hoffart A, Sexton H, Aasland OG, Gude T. A three-year cohort study of the relationships between coping, job stress and burnout after a counselling intervention for help-seeking physicians. BMC Public Health. 2010;10:213.

[32] Viljoen M, Claassen N. Cynicism as subscale of burnout Work. 2017;56(4):499-503.

[33] Zwack J, Schweitzer J. If every fifth physician is affected by burnout, what about the other four? Resilience strategies of experienced physicians. Acad Med. 2013;88(3):382-9.

[34] Sargent MC, Sotile W, Sotile MO, Rubash H, Barrack RL. Quality of life during orthopaedic training and academic practice. Part 1: orthopaedic surgery residents and faculty. J Bone Joint Surg Am. 2009;91(10):2395-405.

[35] Firth-Cozens J. Interventions to improve physicians' wellbeing and patient care. Soc Sci Med. 2001;52(2):215-22. 